\title{
Is there a Diference between Brand and Generic Oxaliplatin?
}

\author{
Kakil I. Rasul ${ }^{1}$, A. Sadig Kamal ${ }^{1}$, Nahla M. Al-Najjar ${ }^{1} \&$ Muhammad H. Oculalan $^{1}$ \\ ${ }^{1}$ Prem Chandra, Al-Amal Hospital/Doha, Qatar \\ Correspondence: Kakil Ibrahim Rasul, CABM, FRCP Edin, ESMO, Senior Consultant Hematology/Oncology, \\ Al-Amal Hospital, P.O. Box 3050, Doha, Qatar. E-mail: kakil954@yahoo.com
}

Received: February 16, 2011 Accepted: March 1, 2012 Online Published: June 18, 2012

doi:10.5539/cco.v1n2p45 URL: http://dx.doi.org/10.5539/cco.v1n2p45

\begin{abstract}
Oxaliplatin-based chemotherapy is the preferred first-line combination chemotherapy for metastatic colorectal cancer. In the last few years the generic oxaliplatin had been introduced, some personal observation that there may be difference in the spectrum of the side effects of this compound generic and the brand preparations. The use of generic drugs has become increasingly common in clinical practice. The generic drugs are chemical medicines have simple and well-defined structures that are manufactured after the patency of the medicine is over. We collected data from two group of patient who had colorectal cancer, $1^{\text {st }}$ group received the brand oxaliplatin based chemotherapy and $2^{\text {nd }}$ group received the generic oxaliplatin based chemotherapy. The hematological adverse effect was not significantly different between the 2 groups but the generic oxaliplatin is associated with higher non hematological side effect than the brand oxaliplatin, both peripheral sensory neuropathy and the hand- foot syndrome. Larger prospective randomised study needed to prove these data.
\end{abstract}

Keywords: oxaliplatin, FOLFOX, colorectal cancer, chemotherapy

\section{Introduction}

In the late 1980s, oxaliplatin (Eloxatin) was found to have activity in advanced CRC, and it is the only platinum derivative with activity against advance colorectal cancer (CRC) (Raymond et al., 1998; Rixe et al., 1996). It is mechanism of action is that it binds and cross-links strands of DNA, forming DNA adducts-thus inhibiting DNA replication and transcription. Oxaliplatin also displays synergistic in vitro cytotoxicity with 5-FU against human colorectal cell lines (de Gramont et al., 2000). A potential mechanism for this synergism is the down regulation of thymidylate synthase by oxaliplatin, which thereby potentiates the efficacy of 5-FU (Fischel et al., 1998; Meyerhardt \& Mayer, 2005). The combination of oxaliplatin plus 5-FU/leucovorin is known as the FOLFOX regimen, and it has become a standard regimen for CRC, both as adjuvant therapy and as treatment for metastatic disease (Meyerhardt \& Mayer, 2005).

Oxaliplatin is a platinum derivative in which the platinum atom is complexed with1, 2 diaminocyclohexane (DACH) and with an oxalate ligand as a leaving group. It was synthesized with the goal of trying to overcome resistance to first- and second generation platinum compounds (Giacchetti et al., 2000). The use of generic drugs has become increasingly common in clinical practice. The generic drugs are chemical medicines have simple and well-defined structures that are manufactured after the patency of the medicine is over. Oxaliplatin is one of the medicine which have both the brand and the generic preparation are available in the market.

The mechanism of action of oxaliplatin is similar to that of cisplatin as well as other platinum compounds. Studies conducted to date indicate that the types and percentages of Pt-DNA adducts formed by oxaliplatin are qualitatively similar to those formed by cisplatin, but preclinical data suggest several unique attributes of the cytotoxic/antitumor activity of oxaliplatin. Oxaliplatin demonstrates a broad spectrum of in vitro cytotoxic and in vivo antitumor activity that differs from that of either cisplatin or carboplatin. Oxaliplatin is active against several cisplatin-resistant cell lines, colon carcinoma, and other solid tumours that are not responsive to cisplatin. In addition, oxaliplatin in combination with 5-FU leads to synergistic antiproliferative activity in several in vivo tumour models (Raymond, Chaney, Taamma, \& Cvitkovic, 1998). Oxaliplatin has been used showed efficacy in treatment of many cancers like colon, gastric, pancreatic, and liver. In phase III studies, oxaliplatin in combination with 5-FU/LV (FOLFOX-4) has demonstrated superiority in terms of response rate and progression-free survival versus 5- FU/LV (De Gramont et al., 2000). Furthermore, the FOLFOX-4 regimen has shown superiority in RR, TTP and OS vs the Saltz regimen of irinotecan in combination with bolus 5-FU/LV. Oxaliplatin has been 
preferentially developed with infusion 5-FU regimens as some safety issues surround its administration with bolus 5-FU/LV (Richard et al., 2004).

\section{Methods and Patients}

A retrospective study for the patients who had colorectal cancer, which had received oxaliplatin containing chemotherapy (Brand) in year 2009 and patients who had colorectal cancer who had received oxaliplatin containing chemotherapy (Generic) in year 2010, and collect the data from the source medical records and medicom data. The data included patient demography stage of the disease and the adverse effect documented for these patients 2 group patients' characteristics are compared and the spectrum of the side effects as well are compared between the 2 groups. Fifty four patients include in each group. Statistical Methods used for Data Analysis, Qualitative and quantitative values were expressed as frequency (percentage) and mean $\pm \mathrm{SD}$. Descriptive statistics were used to summarize baseline demographic and clinical characteristics of the patients. Unpaired' $\mathrm{T}$ test was used to compare means of quantitative measurements between the two independent treatment groups. Chi-square test was used to examine an association of various qualitative measurements between the two treatment groups. In case of having small cell frequency Fisher's exact test was applied to examine the association for variables measured qualitatively between the two groups. P- Value smaller than 0.05 was considered as statistically significant. All statistical analyses were done using statistical packages SPSS 19.0.

\section{Results}

Fifty four patients were included in each group of patients with colorectal cancer received oxaliplatin containing chemotherapy protocol. Statistical analysis revealed that there was no statistically significant difference in patients various baseline demographic and clinical characteristics such as age, gender, nationality, stage of disease, Treatment, and Haematological adverse affects between the Oxaliplatin Brand and Oxaliplatin generic groups ( $\mathrm{p}>$ 0.05).

The percentage of subject having WBC values less than $4(\mathrm{WBC}<4 \mathrm{x} 10)$ was higher in Oxaliplatin Brand group than Oxaliplatin Generic group ( $50 \%$ vs. $35.8 \%$ ). However, this difference was not found to be statistically significant ( $\mathrm{p}=0.139$ ) Though, it was not statistically significant, the percentage of subjects reported side effects as neuropathy, grade 2 and more by clinical assessment was higher in Oxaliplatin Generic group than Oxaliplatin Brand $(11.3 \%$ vs. $7.4 \%$; $\mathrm{p}=0.487)$. Also, other side effect such as Hand-Foot Syndrome was found to be higher in Oxaliplatin Generic group than Oxaliplatin Brand group. But because of the small size study population it doesn't reach statistically significant values (p values 0.139 and 0.487 respectively).

\section{Discussion}

Oxaliplatin is an antineoplastic agent; consists of a platinum atom complexed with 1,2-diaminocyclohexane (DACH) and a labile oxalate ligand. It must undergo nonenzymatic activation before antineoplastic activity occurs.In physiologic solutions, the labile oxalate ligand presumably is displaced, forming several transient reactive complexes (e.g., monoaquo DACH platinum, diaquo DACH platinum). These complexes covalently bind to specific DNA base sequences, producing intrastrand and interstrand DNA cross-links, which are thought to inhibit DNA replication and transcription. Oxaliplatin exhibits antitumor activity against colon carcinoma in vivo and exhibits synergistic antiproliferative activity with fluorouracil (Sanofi-Synthelabo Inc., 2009; Culy, Clemett, \& Wiseman, 2000). Oxaliplatin has been used showed efficacy in treatment of may cancers like colon, gastric, pancreatic, and liver. The use of the FOLFOX regimen as adjuvant treatment for colon cancer was confirmed by the Multicenter International Study of Oxaliplatin/Fluorouracil/Leucovorin in the Adjuvant Treatment of Colon Cancer (MOSAIC). This study concluded that adding oxaliplatin to a regimen of 5-FU and leucovorin improves the adjuvant treatment of colon cancer. The investigators randomly assigned 2,246 patients who had undergone curative resection for stage II or III colon cancer to receive 5-FU alone or with oxaliplatin for 6 months. The primary endpoint was disease-free survival. The result showed that the rate of disease-free survival at 3 years was $78.2 \%$ in the group given 5 -FU/leucovorin plus oxaliplatin vs $72.9 \%$ in the 5 -FU/leucovorin only group $(P=.002)$ (Andre et al., 2004; de Gramont et al., 2003). In metastatic disease, patients treated with FOLFOX had a response rate, time to disease progression, and overall survival time that were superior to those observed with other combination chemotherapies, including IFL (irinotecan plus 5-FU) or IROX (irinotecan plus oxaliplatin). These data support the use of oxaliplatin-based chemotherapy as the preferred first-line combination chemotherapy for metastasis colorectal cancer (de Gramont et al., 2003; Meyerhardt \& Mayer, 2005).

In the last few years the generic oxaliplatin had been introduced, some personal observation that there may be difference in the spectrum of the side effects of this compound generic and the brand preparations. 
Table 1. Patients characteristics

\begin{tabular}{lccc}
\hline Variables & $\begin{array}{c}\text { Oxaliplatin Brand } \\
\text { (N= 54) }\end{array}$ & $\begin{array}{c}\text { Oxaliplatin Generic } \\
\text { (N=53) }\end{array}$ & P-value \\
\hline $\begin{array}{l}\text { Age } \\
\text { Gender }\end{array}$ & $51.46 \pm 10.81$ & $53.06 \pm 11.32$ & 0.484 \\
$\quad$ Male & $34(63.0 \%)$ & $33(62.3 \%)$ & 0.940 \\
$\quad$ Female & $20(37.0 \%)$ & $20(37.7 \%)$ & \\
Nationality & & & \\
$\quad$ Qatari & $15(27.8 \%)$ & $9(17.0 \%)$ & \\
$\quad$ Non Qatari & $39(72.2 \%)$ & $44(83.0 \%)$ & 0.731 \\
Stage of the Disease & & & \\
II & $8(14.8 \%)$ & $9(17.4 \%)$ & \\
III & $20(37.0 \%)$ & $17(32.6 \%)$ & 0.502 \\
IV & $26(48.1 \%)$ & $26(50.0 \%)$ & \\
Treatment & & $27(50.9 \%)$ & \\
Adjuvant & $31(57.4 \%)$ & $26(49.1 \%)$ & \\
Metastatic & $23(42.6 \%)$ & & \\
\hline
\end{tabular}

The use of generic drugs has become increasingly common in clinical practice. The generic drugs are chemical medicines have simple and well-defined structures that are manufactured after the patency of the medicine is over.

Oxaliplatin is one of the medicine which have both the brand and the generic preparation are available in the market. At Al-Amal hospital we have 54 patients with colorectal cancer received chemotherapy brand oxaliplatin based in 2009, and same number in 2010 patients with colorectal cancer who received generic oxaliplatin based chemotherapy. We did retrospective study to review the records of the 2 groups of the patients and find the characteristics of the patients in both group and to compare the spectrum of the toxicities in both groups. Statistical analysis revealed that there was no statistically significant difference in patients various baseline demographic and clinical characteristics such as age, gender, nationality, stage of disease, Treatment, and Haematological adverse affects between the Oxaliplatin Brand and Oxaliplatin generic groups ( $>$ > 0.05) (Table 1).

Table 2. Side effects

\begin{tabular}{|c|c|c|c|}
\hline Variables & $\begin{array}{l}\text { Oxaliplatin Brand } \\
(\mathrm{N}=54)\end{array}$ & $\begin{array}{l}\text { Oxaliplatin Generic } \\
(\mathrm{N}=53)\end{array}$ & P-value \\
\hline \multicolumn{4}{|l|}{ Treatment } \\
\hline Adjuvant & $31(57.4 \%)$ & 27 (50.9 \%) & 0.502 \\
\hline Metastatic & 23 (42.6 \%) & 26 (49.1 \%) & \\
\hline $\begin{array}{l}\text { Hematological S E } \\
\qquad W B C<4 \times 109 / L\end{array}$ & 27 (50.0\%) & 19 (35.8 \%) & 0.139 \\
\hline $\begin{array}{c}\text { Hematological S E } \\
\qquad \mathrm{Hb}<109 / \mathrm{L}\end{array}$ & 7 (13.0 \%) & 8 (15.4%) & 0.721 \\
\hline $\begin{array}{l}\text { Hematological S E } \\
\text { Plt }<140 \times 109 / \mathrm{L}\end{array}$ & 23 (42.6 \%) & 23 (43.4 \%) & 0.933 \\
\hline Neuropathy & $4(7.4 \%)$ & $6(11.3 \%)$ & 0.487 \\
\hline $\begin{array}{l}\text { Other Side Effects- Hand-Foot } \\
\text { Syndrome* }\end{array}$ & $1(1.9 \%)$ & $6(11.3 \%)$ & $0.101 \dagger$ \\
\hline
\end{tabular}

The percentage of subject having WBC values less than 4 (WBC $<4 \times 10$ ) was higher in Oxaliplatin Brand group than Oxaliplatin Generic group (50\% vs. $35.8 \%$ ). However, this difference was not found to be statistically significant ( $p=0.139$ ) Though, it was not statistically significant, the percentage of subjects reported side effects as neuropathy grade 2 and more was higher in Oxaliplatin Generic group than Oxaliplatin Brand (11.3\% vs. 7.4\%; $\mathrm{p}=0.487$ ). The assessment of neuropathy is primarily based on neurologic clinical examination. Also, other side 
effect such as Hand-Foot Syndrome was found to be higher in Oxaliplatin Generic group than Oxaliplatin Brand group. But because of the small size study population it doesn't reach statistically significant values. (P values 0.139 and 0.487 respectively) (Table 2).

These result need to be confirmed in a larger prospective randomised study.

\section{Conclusion}

The generic oxaliplatin is associated with higher side effect than the brand oxaliplatin; both grade 2 and more peripheral sensory neuropathy and the hand- foot syndrome. Larger prospective randomised study needed to prove these data.

\section{Refernces}

Andre, T., Boni, C., Mounedji-Boudiaf, L., Navarro, M., Tabernero, J., Hickish, T., ... de Gramont, A. (2004). Oxalipatin, fluorouracil, and leucovorin as adjuvant treatment for colorectal cancer. N. Engl. J. Med., 350, 2343-2351. http://dx.doi.org/10.1056/NEJMoa032709

Culy, C. R., Clemett, D., \& Wiseman, L. R. (2000). Oxaliplatin: a review of its pharmacological properties and clinical efficacy in metastatic colorectal cancer and its potential in other malignancies. Drugs, 60, 895-924. http://dx.doi.org/10.2165/00003495-200060040-00005

de Gramont, A., Banzi, M., Navarro, M., Taberero, J., Hickish, T., Bridgewater, J., ... Andre, T. (2003). Oxaliplatin/5-FU/LV in adjuvant colon cancer: Result of the international randomized MOSAIC trial (abstract 1015). Proc. Am. Soc. Clin. Oncol., 22, 253.

de Gramont, A., Figer, A., Seymour, M., Homerin, M., Hmissi, A., Cassidy, J., ... Bonetti, A. (2000). Leucovorin and fluorouracil with or without oxaliplatin as first-line treatment in advanced colorectal cancer. J. Clin. Oncol., 18, 2938-2947.

Fischel, J. L., Etienne, M. C., Formento, P., \& Milano, G. (1998). Search for the optimal schedule for the oxaliplatin/5-fluorourail association modulated or not by folinic acid: Precelinical data. Clin. Cancer Res., 4(10), 2529-35.

Giacchetti, S., Perpoint, B., Zidani, R., Le Bail, N., Faggiuolo, R., Focan, C., ... Lévi, F. (2000). Phase III multicenter randomized trial of oxaliplatin added to chronomodulated fluorouracil-leucovorin as first-line treatment of metastatic colorectal cancer. J. Clin. Oncol., 18, 136-147.

Goldberg, R. M., Morton, R. F., Sargent, D. J., et al. (2003). N9741: oxaliplatin (oxal) or CPT-11 + fluorouracil (5FU)/leucovorin (LV) or oxal + CPT-11 in advanced colorectal cancer (CRC). Updated efficacy and quality of life (QOL) data from an intergroup study. Paper presented at: American Society of Clinical Oncology Annual Meeting; June 1, Chicago, Ill.

Goldberg, R. M., Sagent, D. J., Morton, R. F., Fuchs, C. S., Ramanathan, R. K., Williamson, S. K., ... Alberts, S. R. (2004). A randomized controlled trial of fluorouracil plus leucovorin, irinotecan, and oxaliplatin combinations in patients with previously untreated colorectal cancer. J. Clin. Oncol., 22, 23-30. http://dx.doi.org/10.1200/JCO.2004.09.046

Meyerhardt, J. A., \& Mayer, R. J. (2005). Systemic therapy for colorectal cancer. N. Engl. J. Med., 352, 476-487. http://dx.doi.org/10.1056/NEJMra040958

Raymond, E., Chaney, S. G., Taamma, A., \& Cvitkovic, E. (1998). Oxaliplatin a review of preclinical and clinical studies. Ann. Oncol., 9(10), 1053-1071. http://dx.doi.org/10.1023/A:1008213732429

Raymond, E., Faivre, S., Woynarowski, J. M., \& Chaney, S. G. (1998). Oxaliplatin: Mechanism of action and antineoplastic activity. Semin Oncol, 25, 4-12.

Rixe, O., Ortuzar, W., Alvarez, M., Parker, R., Reed, E., Paull, K., \& Fojo, T. (1996). Oxaliplatin, tetraplatin, cisplatin, and carboplatin: spectrum of activity in drug-resistant cell lines and in the cell lines of the National Cancer Institutes Anticancer Drug Screen panel. Biochem. Pharmacol., 52, 1855-1865. http://dx.doi.org/10.1016/S0006-2952(97)81490-6

Sanofi-Synthelabo Inc. (2009 Mar). Eloxatin (oxaliplatin) for injection prescribing information. New York. 